\title{
Elevated levels of kynurenic acid in the cerebrospinal fluid of patients with bipolar disorder
}

\author{
Sara K. Olsson, MS; Martin Samuelsson, MD; Peter Saetre, PhD; Leif Lindström, MD, PhD; \\ Erik G. Jönsson, MD, PhD; Conny Nordin, MD, PhD; Göran Engberg, PhD; \\ Sophie Erhardt, PhD; Mikael Landén, MD, PhD
}

Olsson, Engberg, Erhardt - Department of Physiology and Pharmacology, Karolinska Institutet, Stockholm; Samuelsson, Nordin - Department of Clinical and Experimental Medicine, Section of Psychiatry, Faculty of Health Sciences, Linköping University, Linköping; Saetre, Jönsson - Department of Clinical Neuroscience, HUBIN project, Karolinska Institutet and Hospital, Stockholm; Lindström — Department of Neuroscience, Section of Psychiatry, Uppsala University, Uppsala; Landén — Department of Clinical Neuroscience, Karolinska Institutet, Stockholm, and the Institute of Neuroscience and Physiology, University of Gothenburg, Gothenburg, Sweden

\begin{abstract}
Background: Patients with schizophrenia show elevated brain levels of the neuroactive tryptophan metabolite kynurenic acid (KYNA). This astrocyte-derived mediator acts as a neuroprotectant and modulates sensory gating and cognitive function. We measured the levels of KYNA in the cerebrospinal fluid (CSF) of patients with bipolar disorder and healthy volunteers to investigate the putative involvement of KYNA in bipolar disorder. Methods: We obtained CSF by lumbar puncture from 23 healthy men and 31 euthymic men with bipolar disorder. We analyzed the samples using high-performance liquid chromatography. Results: Patients with bipolar disorder had increased levels of KYNA in their CSF compared with healthy volunteers (1.71 nM, standard error of the mean [SEM] 0.13 v. 1.13 nM, SEM 0.09; $p=0.002$. The levels of KYNA were positively correlated with age among bipolar patients but not healthy volunteers. Limitations: The influence of ongoing drug treatment among patients cannot be ruled out. We conducted our study during the euthymic phase of the disease. Conclusion: Brain KYNA levels are increased in euthymic men with bipolar disorder. In addition, KYNA levels increased with age in these patients. These findings indicate shared mechanisms between bipolar disorder and schizophrenia. Elevated levels of brain KYNA may provide further insight to the pathophysiology and progression of bipolar disorder.
\end{abstract}

\section{Introduction}

Bipolar disorder is characterized by recurrent episodes of mania and depression, interspersed with euthymic periods when depressive or manic symptoms are absent. Severe manic episodes often feature psychotic symptoms, such as hallucinations and delusions that may be indistinguishable from acute psychosis in schizophrenia. Cognitive impairments of executive function, attention and memory are evident in all states of bipolar disorder, ${ }^{1,2}$ and cognitive deficits are also regarded as core symptoms in schizophrenia. These and other similarities in clinical characteristics are indicative of a partially shared pathophysiology of these disorders. Indeed, several common susceptibility genes have been identi- fied, ${ }^{3}$ and a recent population study including more than 2 million Swedish families revealed a substantial genetic association between schizophrenia and bipolar disorder. ${ }^{4}$ Despite much effort, our knowledge about the causes and underlying mechanisms of bipolar disorder and related psychiatric disorders is still unsatisfactory. Although excessive dopamine activity is proposed in the manic state, ${ }^{5}$ no biological markers have yet been identified that correspond with the disease.

Kynurenic acid (KYNA) is a tryptophan metabolite of the kynurenine pathway that acts as an endogenous antagonist on both the glycine site of the N-methyl-D-aspartate ${ }^{6,7}$ receptor and on the nicotinergic $\alpha 7^{*}$ acetylcholine receptor. ${ }^{8}$ The compound is synthesized in and released by astrocytes in the

Correspondence to: Dr. S. Erhardt, Department of Physiology and Pharmacology, Karolinska Institutet, SE-171 77 Stockholm, Sweden; fax 4683106 22; sophie.erhardt@ ki.se

J Psychiatry Neurosci 2010;35(3):195-9.

Submitted Dec. 10, 2009; Revised Feb. 2, 2010; Accepted Feb. 2, 2010.

DOI: 10.1503/jpn.090180 
brain., ${ }^{9,10}$ Elevated levels of KYNA have previously been observed in patients with schizophrenia, both in the cerebrospinal fluid (CSF) $)^{11,12}$ and postmortem prefrontal cortex. ${ }^{13}$ Interestingly, KYNA tonically modulates midbrain dopamine activity, ${ }^{14-20}$ indicating a potential role of this compound in dopamine-related diseases. ${ }^{21}$

Our objective was to test the hypothesis that KYNA plays a role in the pathophysiology of bipolar disorder, by analyzing the concentration of KYNA in the CSF of patients with bipolar disorder and controls.

\section{Methods}

\section{Participants}

We recruited patients from December 2005 to April 2008 from a long-term follow-up program at a bipolar outpatient unit at the Northern Stockholm psychiatric clinic, Stockholm, Sweden. Consecutive new outpatients referred for treatment and continuing patients at the bipolar outpatient unit were invited to participate, provided that they were at least 18 years old and met the DSM-IV criteria for bipolar disorder type I or II.

A complete account of the clinical investigation procedure has been previously published..$^{22}$ Briefly, the clinical diagnosis of bipolar disorder was established according to the Affective Disorder Evaluation, ${ }^{22}$ which was previously used in the STEP-BD project. ${ }^{23}$ With the permission of its originator Gary S. Sachs, the ADE was translated and modified to suit Swedish conditions. To minimize interrater variability, the collected information was presented at a diagnostic caseconference, and a consensus panel of experienced boardcertified psychiatrists who specialize in bipolar disorder made the final diagnostic decision at this conference.

We collected CSF samples when the patients were symptom free and in a stable euthymic mood, as judged by a physician. For ethical reasons, the patients continued to take their prescribed medication.

We recruited 36 healthy male volunteers from among medical students, hospital staff members and their relatives. They all underwent a medical check-up including laboratory tests (electrolytes, blood, thyroid, kidney and liver) and a physical examination. The volunteers had to have been free of medication for at least 1 month and free from any form of substance abuse. We included those who smoked or consumed coffee.

The volunteers underwent a semistructured interview using the Structured Clinical Interview for DSM-IV Axis I disorders (SCID-I). ${ }^{24}$ The interview was directed toward affective disorders, anxiety disorders and drug abuse. The volunteers also completed the SCID-II questionnaire for personality disorders. ${ }^{25}$ We considered 30 healthy volunteers to be eligible for inclusion in the study with respect to the clinical interview and SCID results. All were considered healthy by the psychiatrist performing the examinations and showed no signs of psychiatric or somatic illness or had any laboratory test results outside of the standardized reference ranges. None of the volunteers had a family history of major psychosis or suicide in first- or second-degree relatives.

This study was carried out in accordance with the Declara- tion of Helsinki for experiments involving humans. All patients and healthy volunteers received verbal and written information and gave their written informed consent. Patients were excluded if they were unable to complete the standard clinical assessment or were incapable of providing informed consent. The study was approved by the Ethical Committees of the University Hospital in Linköping, the Karolinska Institutet and the Medical Faculty of Uppsala University, Sweden.

\section{Analysis of KYNA}

We drew CSF from the L4-L5 interspace between 9 and $11 \mathrm{am}$ after a night of fasting and bed rest. In total, $12 \mathrm{~mL} \mathrm{CSF}$ was collected, inverted to avoid gradient effects, divided into aliquots and frozen at $-870^{\circ} \mathrm{C}$ until assayed. Data from a subset of the control samples have previously been published. ${ }^{12}$

We performed the analysis of KYNA using an isocratic reversed-phase high-performance liquid chromatography (HPLC) system, including a dual-piston, high-liquid delivery pump (Bischoff Chromatography), a ReproSil-Pur C18 column $(4 \times 150 \mathrm{~mm}$, Dr. Maisch $\mathrm{GmbH})$ and a fluorescence detector (Jasco Ltd.) with an excitation wavelength of $344 \mathrm{~nm}$ and an emission wavelength of $398 \mathrm{~nm}$ (18 nm bandwidth), essentially as previously described. ${ }^{26}$ A mobile phase of $50 \mathrm{mM}$ sodium acetate ( $\mathrm{pH} 6.2$, adjusted with acetic acid) and $7.0 \%$ acetonitrile was pumped through the reversed-phase column at a flow rate of $0.5 \mathrm{~mL} / \mathrm{min}$. Samples of $50 \mu \mathrm{L}$ were manually injected (Ecom). Zinc acetate ( $0.5 \mathrm{M}$ not $\mathrm{pH}$ adjusted) was delivered after the column by a peristaltic pump (P-500; Pharmacia) at a flow rate of $0.10 \mathrm{~mL} / \mathrm{min}$. Signals from the fluorescence detector were transferred to a computer for analysis with Datalys Azur (version 4.6.0.0; http://datalys.net). The retention time of KYNA was about 7-8 minutes. Initially, the sensitivity of the system was verified by analysis of a standard mixture of KYNA with concentrations from 0.5 to $30 \mathrm{nM}$, which resulted in a linear standard plot. To verify the reliability of this method, some samples were analyzed in duplicate, and the mean intraindividual variation was below $5 \%$.

\section{Statistical analysis}

The KYNA values are given as mean and standard errors of the mean (SEM). Differences in the levels of KYNA were established using the Mann-Whitney $U$ test. We performed linear regression analysis using the least squares method to study the relation between KYNA level and age. We tested for a normal distribution of residuals with the Anderson-Darling test for each of the groups and none of the groups deviated from normality $(p>0.25)$. We assumed significance for all values with $p<0.05$.

\section{Results}

We included 31 men with bipolar disorder and 23 healthy male volunteers. The mean age of the men with bipolar disorder was 36.3 (standard deviation [SD] 9.2, range 20-52 yr), and the mean age of the healthy men was 33.1 (SD 6.9, range 24-51 yr). Of the included men, 18 met the DSM-IV criteria 
for bipolar disorder type I (mean age 37.3, SD $10.5 \mathrm{yr}$ ) and 13 met the criteria for type II (mean age 34.8, SD $7.1 \mathrm{yr}$ ). Because there were a limited number of female healthy volunteers, we did not include female patients in this study.

All but 2 patients with bipolar disorder were taking medication at the time of sampling. The most commonly used treatments were as follows: lithium $(n=21)$, lamotrigine $(n=$ $7)$, quetiapine $(n=5)$, mirtazapine $(n=4)$, propiomazine $(n=$ $4)$ and valproate $(n=3)$. Some of these patients were taking more than one drug: lithium and lamotrigine $(n=5)$ lithium and propiomazine $(n=3)$, lithium and mirtazapine $(n=2)$, lithium, lamotrigine and quetiapine $(n=2)$, lamotrigine and valproate $(n=1)$, valproate and quetiapine $(n=1)$, valproate and mirtazapine $(n=1)$, lamotrigine and propiomazine $(n=$ $1)$, lithium, lamotrigine and mirtazapine $(n=1)$ and lithium, quetiapine and propiomazine $(n=1)$.

The KYNA levels in the CSF of patients with bipolar disorder were significantly higher (1.71 nM, SEM $0.13 \mathrm{nM}$, range $0.45-3.19 \mathrm{nM}, n=31)$ than in the healthy volunteers $(1.13 \mathrm{nM}$, SEM $0.09 \mathrm{nM}$, range $0.34-2.17 \mathrm{nM}, n=23, U=181, p=0.002$, Fig. 1). There was no difference in KYNA levels between patients with bipolar disorder I and II (type I: $1.77 \mathrm{nM}$, SEM 0.17 $\mathrm{nM}, n=18$; type II: $1.63 \mathrm{nM}$, SEM $0.21 \mathrm{nM}, n=13)$. The level of KYNA was significantly correlated with age in patients $(r=$ $0.49, p=0.005, \mathrm{y}=0.0397 \mathrm{x}+0.274)$ but not in healthy volunteers $(r=0.23, p=0.29, \mathrm{y}=0.0135 \mathrm{x}+0.686$; Fig. 2$)$.

\section{Discussion}

We found that KYNA levels in the CSF were higher in euthymic men with bipolar disorder than in healthy men. Furthermore, KYNA levels were positively correlated with age in the patient group. These results are similar to our previous studies, in which we found increased levels of KYNA in the CSF of patients with schizophrenia. ${ }^{11,12}$ In the present study, there was no correlation between age and KYNA level in healthy men. An age-related increase in KYNA concentration has previously been reported in rats ${ }^{27,28}$ but not in healthy volunteers. ${ }^{11,12,29-31}$ Because our samples were limited by a rather narrow age range (20-52 yr), we cannot ruled out that brain KYNA may increase with age in older healthy individuals.

Kynurenic acid is an astrocyte-derived antagonist that inhibits brain glutamatergic and cholinergic transmission. The functional significance of the observed elevation in brain KYNA levels (about 50\%) is unclear. Notably though, experimental studies reported that elevations of KYNA in the rat brain, within the same order of magnitude, are associated with a marked activation of the firing of midbrain dopamine neurons. ${ }^{14-20}$ Thus, the elevation of brain KYNA in bipolar disorder likely functionally influences glutamatergic, cholinergic and dopaminergic neurotransmission in these patients.

\section{Limitations}

A major limitation of our study is that most of the patients were taking psychotropic drugs during CSF sampling. However, several animal studies have shown that some of the drugs used by the patients, such as valproate and lamotrigine, do not affect brain KYNA concentration when administered to animals at clinically relevant concentrations. ${ }^{32}$ Further, chronic treatment with antipsychotic drugs reduces brain KYNA levels in rats,,$^{33}$ a finding also supported by postmortem findings in patients with schizophrenia, ${ }^{34}$ thus arguing against an influence of these drugs on the present results. Although we did not perform a complete correlation analysis between KYNA levels and medication status, KYNA levels did not differ between

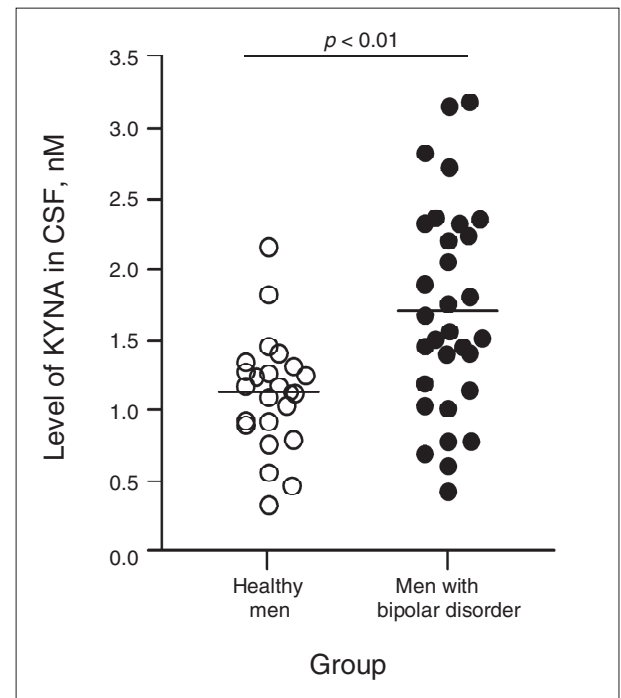

Fig. 1: Kynurenic acid (KYNA) in the cerebrospinal fluid (CSF) of healthy men and men with bipolar disorder. Each point represents the concentration of KYNA in a single CSF sample. The mean of each group is indicated by the line. Mann-Whitney $U$ test, $U=181$.

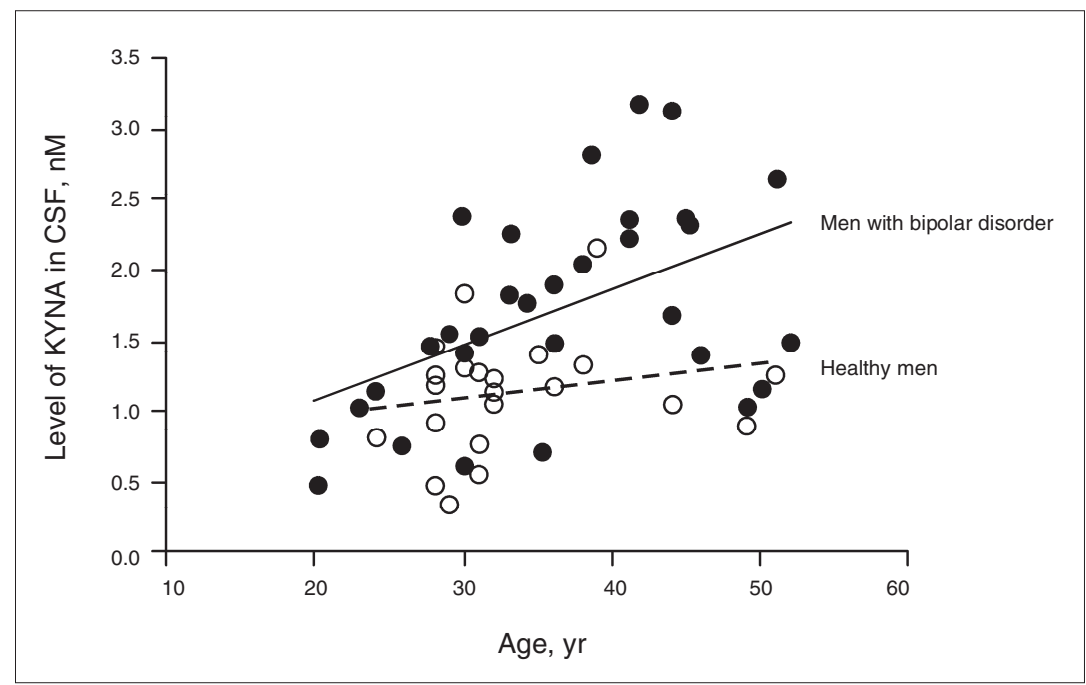

Fig. 2: Linear regression analysis of the amount of kynurenic acid (KYNA) in the cerebrospinal fluid (CSF) and age of healthy men (open circles) and in men with bipolar disorder (closed circles). A significant correlation between KYNA and age was observed in male patients with bipolar disorder $(r=0.49, p=0.005, \mathrm{y}=0.0397 \mathrm{x}+0.274)$. 
patients taking antipsychotic drugs or lithium as compared with the rest of the group (data not shown). The observation that KYNA in CSF increases with age, despite similar medication doses given to all patients regardless of age, further argues against any direct influence of ongoing medication. Taken together, the observed elevation of KYNA in the CSF of bipolar patients is more likely the result of underlying processes that might be linked to the progression of the disease. Our findings are in agreement with those of a postmortem study of patients with bipolar disorder showing increased levels of brain kynurenine, the immediate precursor of KYNA, and increased expression of the tryptophan-degrading enzyme tryptophan 2,3-dioxygenase (TDO2). ${ }^{34}$

The observed elevation of KYNA levels in the CSF of bipolar patients is important in light of the fact that cognitive dysfunctions are present in all states of the disease, including the euthymic state. Furthermore, these dysfunctions are known to progress with the course and severity of the disease. ${ }^{35-37}$ Numerous studies have shown an intimate relation between cognitive function and glutamatergic and cholinergic neurotransmission. ${ }^{38,39}$ Indeed, a large body of studies has demonstrated that KYNA is involved in the modulation of behaviour and cognition. Thus, in rodents, pharmacologically increased levels of KYNA have been found to disrupt prepulse inhibition,,$^{40}$ a feature also observed in patients with bipolar disorder during both acute psychotic mania ${ }^{41}$ and euthymia, ${ }^{42}$ as well as in patients with schizophrenia. ${ }^{43}$ Furthermore, pharmacologically elevated KYNA levels impair contextual learning and working memory in rats. ${ }^{44,45}$ Accordingly, kynurenine aminotransferase II knockout mice, which have low levels of endogenous KYNA, show increased performance in passive avoidance T-maze tests. ${ }^{46,47}$ Taken together, these findings suggest that KYNA might causally contribute to the development of the decline in cognitive function during the progression of bipolar disorder.

\section{Conclusion}

Men with bipolar disorder have increased levels of KYNA in their CSF compared with healthy men. Because experimental studies have described the important role of KYNA in learning and memory, ${ }^{21}$ KYNA may be involved in the mechanisms leading to cognitive deficits in bipolar disorder.

Acknowledgements: We dedicate this article to the memory of our dear friend Conny Nordin, who died July 5, 2008. We thank the health professionals who facilitated this study. In particular, we thank study coordinator Mrs. Martina Wennberg and research nurses Mrs. Agneta Carswärd-Kjellin, Mrs. Stina Stadler, Mrs. Hazel Holmberg-Forsyth and Mrs. Margareta Krona. We also thank Dr. Christian Johansson.

Funding: Financial support for this study was provided through the regional agreement on medical training and clinical research between Stockholm County Council and the Karolinska Institutet, and through grants from the Hållstens Forskningsstiftelse, Swedish Brain Foundation, the Östergötland County Council, Svenska Läkaresällskapet, Karolinska Institutet, Torsten och Ragnar Söderbergs stiftelse and the Swedish Research Council (no. 2009-4046 to S.E., 2008-3822 and 20093068 to G.E., K2008-62x-14647-06-3 to M.L., and 2006-986 and 20062992 to E.G.J.). No funding sources had any role in study design, in the collection, analysis and interpretation of data, in the writing of the report, or in the decision to submit the paper for publication.

Competing interests: None declared.

Contributors: Drs. Engberg, Erhardt, Nordin and Landén designed the study. Drs. Samuelsson, Lindström, Jönsson, Nordin and Landén acquired the data, which Drs. Saetre, Erhardt, Nordin and Landén and Ms. Olsson analyzed. Drs. Saetre, Engberg, Erhardt, Nordin and Landén and Ms. Olsson wrote the article. All authors reviewed the article and approved its publication.

\section{References}

1. Bearden CE, Hoffman KM, Cannon TD. The neuropsychology and neuroanatomy of bipolar affective disorder: a critical review. Bipolar Disord 2001;3:106-50, discussion 151-3.

2. Martínez-Arán A, Vieta E, Reinares M, et al. Cognitive function across manic or hypomanic, depressed, and euthymic states in bipolar disorder. Am J Psychiatry 2004;161:262-70.

3. Miklowitz DJ, Johnson SL. The psychopathology and treatment of bipolar disorder. Annu Rev Clin Psychol 2006;2:199-235.

4. Lichtenstein P, Yip BH, Bjork C, et al. Common genetic determinants of schizophrenia and bipolar disorder in Swedish families: a population-based study. Lancet 2009;373:234-9.

5. Berk M, Dodd S, Kauer-Sant'anna M, et al. Dopamine dysregulation syndrome: implications for a dopamine hypothesis of bipolar disorder. Acta Psychiatr Scand Suppl 2007;434:41-9.

6. Birch PJ, Grossman CJ, Hayes AG. Kynurenic acid antagonises responses to NMDA via an action at the strychnine-insensitive glycine receptor. Eur J Pharmacol 1988;154:85-7.

7. Parsons CG, Danysz W, Quack G, et al. Novel systemically active antagonists of the glycine site of the N-methyl-D-aspartate receptor: electrophysiological, biochemical and behavioral characterization. J Pharmacol Exp Ther 1997;283:1264-75.

8. Hilmas C, Pereira EF, Alkondon M, et al. The brain metabolite kynurenic acid inhibits alpha7 nicotinic receptor activity and increases non-alpha7 nicotinic receptor expression: physiopathological implications. J Neurosci 2001;21:7463-73.

9. Swartz KJ, During MJ, Freese A, et al. Cerebral synthesis and release of kynurenic acid: an endogenous antagonist of excitatory amino acid receptors. J Neurosci 1990;10:2965-73.

10. Gramsbergen JB, Hodgkins PS, Rassoulpour A, et al. Brain-specific modulation of kynurenic acid synthesis in the rat. J Neurochem 1997;69:290-8.

11. Erhardt S, Blennow K, Nordin C, et al. Kynurenic acid levels are elevated in the cerebrospinal fluid of patients with schizophrenia. Neurosci Lett 2001;313:96-8.

12. Nilsson LK, Linderholm KR, Engberg G, et al. Elevated levels of kynurenic acid in the cerebrospinal fluid of male patients with schizophrenia. Schizophr Res 2005;80:315-22.

13. Schwarcz R, Rassoulpour $\mathrm{A}, \mathrm{Wu} \mathrm{HQ}$, et al. Increased cortical kynurenate content in schizophrenia. Biol Psychiatry 2001;50:521-30.

14. Erhardt S, Öberg H, Mathe JM, et al. Pharmacological elevation of endogenous kynurenic acid levels activates nigral dopamine neurons. Amino Acids 2001;20:353-62.

15. Erhardt S, Engberg G. Increased phasic activity of dopaminergic neurones in the rat ventral tegmental area following pharmacologically elevated levels of endogenous kynurenic acid. Acta Physiol Scand 2002;175:45-53.

16. Nilsson LK, Linderholm KR, Erhardt S. Subchronic treatment with kynurenine and probenecid: effects on prepulse inhibition and firing of midbrain dopamine neurons. J Neural Transm 2006;113:557-71.

17. Schwieler L, Erhardt S, Nilsson L, et al. Effects of COX-1 and COX-2 inhibitors on the firing of rat midbrain dopaminergic neurons possible involvement of endogenous kynurenic acid. Synapse 2006; 59:290-8. 
18. Schwieler L, Linderholm KR, Nilsson-Todd LK, et al. Clozapine interacts with the glycine site of the NMDA receptor: electrophysiological studies of dopamine neurons in the rat ventral tegmental area. Life Sci 2008;83:170-5.

19. Linderholm KR, Andersson A, Olsson S, et al. Activation of rat ventral tegmental area dopamine neurons by endogenous kynurenic acid: a pharmacological analysis. Neuropharmacology 2007;53:918-24.

20. Olsson SK, Andersson AS, Linderholm KR, et al. Elevated levels of kynurenic acid change the dopaminergic response to amphetamine: implications for schizophrenia. Int J Neuropsychopharmacol 2009;12:501-12.

21. Erhardt S, Olsson SK, Engberg G. Pharmacological manipulation of kynurenic acid: potential in the treatment of psychiatric disorders. CNS Drugs 2009;23:91-101.

22. Rydén E, Thase ME, Straht D, et al. A history of childhood attention-deficit hyperactivity disorder (ADHD) impacts clinical outcome in adult bipolar patients regardless of current ADHD. Acta Psychiatr Scand 2009;120:239-46.

23. Sachs GS, Thase ME, Otto MW, et al. Rationale, design, and methods of the systematic treatment enhancement program for bipolar disorder (STEP-BD). Biol Psychiatry 2003;53:1028-42.

24. First MB, Gibbon M, Spitzer RL, et al. Structured Clinical Interview for DMS-IV Axis I Disorders (SCID-I), Clinician Version. Washington (DC): American Psychiatric Press; 1997.

25. First MB, Gibbon M, Spitzer RL, et al. Structured Clinical Interview for DMS-IV Axis II Personality Disorders (SCID-II). Washington (DC): American Psychiatric Press; 1997.

26. Swartz KJ, Matson WR, MacGarvey U, et al. Measurement of kynurenic acid in mammalian brain extracts and cerebrospinal fluid by high-performance liquid chromatography with fluorometric and coulometric electrode array detection. Anal Biochem 1990;185:363-76.

27. Moroni F, Russi P, Carlá V, et al. Kynurenic acid is present in the rat brain and its content increases during development and aging processes. Neurosci Lett 1988;94:145-50.

28. Gramsbergen JB, Schmidt W, Turski WA, et al. Age-related changes in kynurenic acid production in rat brain. Brain Res 1992;588:1-5.

29. Heyes MP, Saito K, Crowley JS, et al. Quinolinic acid and kynurenine pathway metabolism in inflammatory and non-inflammatory neurological disease. Brain 1992;115:1249-73.

30. Atlas A, Gisslén M, Nordin C, et al. Acute psychotic symptoms in HIV-1 infected patients are associated with increased levels of kynurenic acid in cerebrospinal fluid. Brain Behav Immun 2007;21: 86-91.

31. Nilsson LK, Nordin C, Jönsson EG, et al. Cerebrospinal fluid kynurenic acid in male and female controls - correlation with monoamine metabolites and influences of confounding factors. J Psychiatr Res 2007;41:144-51.

32. Kocki T, Wielosz M, Turski WA, et al. Enhancement of brain kynurenic acid production by anticonvulsants - Novel mechanism of antiepileptic activity? Eur J Pharmacol 2006;541:147-51.

33. Ceresoli-Borroni G, Rassoulpour A, Wu HQ, et al. Chronic neuroleptic treatment reduces endogenous kynurenic acid levels in rat brain. J Neural Transm 2006;113:1355-65.

34. Miller CL, Llenos IC, Dulay JR, et al. Upregulation of the initiating step of the kynurenine pathway in postmortem anterior cingulate cortex from individuals with schizophrenia and bipolar disorder. Brain Res 2006;1073-1074:25-37.

35. van Gorp WG, Altshuler L, Theberge DC, et al. Cognitive impairment in euthymic bipolar patients with and without prior alcohol dependence. A preliminary study. Arch Gen Psychiatry 1998;55:41-6.

36. Cavanagh JT, Van Beck M, Muir W, et al. Case-control study of neurocognitive function in euthymic patients with bipolar disorder: an association with mania. Br J Psychiatry 2002;180:320-6.

37. Clark L, Iversen SD, Goodwin GM. Sustained attention deficit in bipolar disorder. Br J Psychiatry 2002;180:313-9.

38. Robbins TW, Murphy ER. Behavioural pharmacology: 40+ years of progress, with a focus on glutamate receptors and cognition. Trends Pharmacol Sci 2006;27:141-8.
39. Levin ED, McClernon FJ, Rezvani AH. Nicotinic effects on cognitive function: behavioral characterization, pharmacological specification, and anatomic localization. Psychopharmacology (Berl) 2006;184:523-39.

40. Erhardt S, Schwieler L, Emanuelsson C, et al. Endogenous kynurenic acid disrupts prepulse inhibition. Biol Psychiatry 2004;56:255-60.

41. Perry W, Minassian A, Feifel D, et al. Sensorimotor gating deficits in bipolar disorder patients with acute psychotic mania. Biol Psychiatry 2001;50:418-24.

42. Gogos A, van den Buuse M, Rossell S. Gender differences in prepulse inhibition (PPI) in bipolar disorder: men have reduced PPI, women have increased PPI. Int J Neuropsychopharmacol 2009;12:1249-59.

43. Geyer MA, Braff DL. Startle habituation and sensorimotor gating in schizophrenia and related animal models. Schizophr Bull 1987;13: 643-68.

44. Chess AC, Bucci DJ. Increased concentration of cerebral kynurenic acid alters stimulus processing and conditioned responding. Behav Brain Res 2006;170:326-32.

45. Chess AC, Simoni MK, Alling TE, et al. Elevations of endogenous kynurenic acid produce spatial working memory deficits. Schizophr Bull 2007;33:797-804.

46. Potter MC, Elmer GI, Guidetti P, et al. Improved behavioural performance of kynurenine aminotransferrase II-deficient mice in hippocampus-based learning and memory paradigms [abstract no. 2659]. In: Neuroscience Meeting Planner. San Diego (CA): Society for Neuroscience; 2006.

47. Bergeron R, Wu H-Q, Potter MC, et al. Mice with reduced brain kynurenic acid levels have high extracellular glutamate and show enhanced LTP in the hippocampus [abstract no. 80715]. In: Neuroscience Meeting Planner. San Diego (CA): Society for Neuroscience; 2007.

\section{In $\mathrm{MDD}$, reaching the therapeutic dose is important.}

\section{But do you aim for it right from the start?}

*Major depressive disorder

Wyeth

(c) 2010 Wyeth Canada, owner, now a part of Pfizer Inc. Member Montreal, Canada H4R 1 J6 